\section{Ajuste de un modelo para estimar el contenido de humedad del suelo utilizando TVDI en el Estado de Sinaloa, México*}

\section{Adjusting a model to estimate humidity content of soil by using TVDI in the State of Sinaloa, Mexico}

\author{
Miguel Armando, López-Beltrán** Miguel López Meza***, \\ Martín Abraham, Tirado Ramírez, ****
}

\begin{abstract}
**Maestro en Ciencias, Facultad de Agronomía, Universidad Autónoma de Sinaloa. E-mail: miguel.armandolb@gmail.com. Dirección: Km.17.5, Carretera Culiacán - El Dorado, c.p. 80000 , Culiacán, Sinaloa, México.

*** Doctor en Ciencias, Facultad de Agronomía, Universidad Autónoma de Sinaloa.

****Maestro en Ciencias, Facultad de Agronomía, Universidad Autónoma de Sinaloa.
\end{abstract}

\section{Resumen}

Para la estimación del contenido de humedad en el suelo, existen diversos métodos entre los cuales son directos e indirectos. En percepción remota o teledetección, se puede utilizar el TVDI (Temperature-Vegetation Dryness Index), que utiliza de parámetros de entrada las temperaturas superficiales terrestres e índices de vegetación. La propuesta de este trabajo, es presentar una manera rápida de reajustar aquellos valores donde los cuerpos de agua presentan algún grado de sequedad en el suelo para toda un área de estudio. Se seleccionaron 4 años (20003, 2006, 2009 y 2012), se estimó el TVDI con insumos de productos Modis. Se propuso un valor de ajuste basado en la diferencia de la media y desviación estándar de los cuerpos de agua, se aplica este valor en el producto generado dando como resultado que los cuerpos de agua presentan ahora si una saturación de humedad en sus suelos. Los valores de ajustes son diferentes para cada año de estudio. El contenido de humedad es consistente a la variabilidad esperada acorde a los diferentes usos de suelo y vegetación. Los resultados están acorde a los reportes de sequía reportadas para el Estado de Sinaloa.

Palabra clave: TVDI, Modis, SIG, Percepción Remota, agua, ajuste modelo.

\begin{abstract}
There are various direct and indirect methods used to estimate soil humidity. In remote perception or tele-detection, the TVDI, (Temperature-Vegetation Dryness Index), may be used, which uses terrestrial surface temperatures, and vegetation indexes as input
\end{abstract}

Recibido: $11 / 01 / 2016$

Revisado: 21/02/2016

Aceptado: 10/12/2016

Correspondencia de autor: miguel.armandolb@gmail.com

(C) 2016 Universidad La Gran Colombia. Este es un artículo de acceso abierto, distribuido bajo los términos de la licencia Creative Commons Attribution License, que permite el uso ilimitado, distribución y reproducción en cualquier medio, siempre que el autor original y la fuente se acrediten.

1 Surge en la necesidad de mejorar las variables de entrada en un modelo de identificación a desertificación de la tesis "Integración de imágenes del sensor MODIS y cartografía temática para la simulación de modelos geoespaciales para obtener zonas propensas a desertificación en el Estado de Sinaloa, México".

Cómo citar:

López-B.M.,López, M.M.,Tirado,R.M.(2016) Ajuste de un modelo para estimar el contenido de humedad del suelo utilizando TVDI en el Estado de Sinaloa, México. UGCiencia 22, 25-36

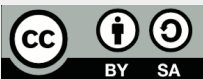


standards. The proposal of this work is to present a quick manner of readjusting those figures where water bodies undergo some dry degree of the soil for the whole area of study. Four years were selected (2003, 2006, 2009 and 2012), the TVDI was estimated with input of MODIS products. An adjustment value was proposed based on the difference of the mean, and standard deviation of water bodies; this figure is applied to the resulting product, which lead to establish that water bodies show a humidity saturation of their soil. Adjustment values are different for each year of study. Humidity content is consistent to expected variability according to the various uses of soil and vegetation. Results agree with drought reports to the State of Sinaloa.

Key words: TVDI, MODIS, SIG, Remote perception, water, model adjustment.

\section{Introducción}

Existen diversos estudios que han mostrado variaciones en el clima, evidenciando que en el mundo se está calentando y registrando aumentos en la temperatura global tanto en la superficie terrestre como a nivel del mar. Conforme a la variación al contenido de calor en distintas cubiertas terrestres y los cambios en las precipitaciones catalizan los procesos de cambio climático (IPCC, 2008).

Aproximadamente, la cobertura denominadas tierras secas en la superficie terrestre es alrededor de $45 \%$, donde el $35 \%$ de la población vive en estas zonas (García, Fernández, Villagarcía, Puigdefábregas y Sandholt, 2014). En la década del 2000, se empezó a orientar la investigación hacia usos eficientes en los recursos hídricos, ya sea para subsistir o intercambiar bienes o servicios; puesto que el mayor volumen de agua lo ocupa el uso agrícola de riego (Conagua, 2011), por lo que es necesario considerar el contenido de humedad del suelo, ya que es un parámetro importante utilizado en diversas investigaciones de diferentes campos tales como meteorología, hidrología y agricultura (Han, Wang y Zhao, 2010; Chen, Chen, Son y Chang, 2011). Las variaciones en el contenido de humedad producen cambios en la superficie en el balance energético, escorrentías, y producción vegetativa (Han et al., 2010).
En cualquier modelo geoespacial se requiere una serie de parámetros y variables que simulen el comportamiento del fenómeno a estudiar. En percepción remota, algunos parámetros se pueden calcular para estimar la humedad en el suelo a partir de los valores contenidos en el espectro electromagnético y aplicados a diversos métodos de estimación (Pertovt, Rivas, Juliano, Osmar y Vives, 2008). Por otro lado, obtener una precisión de los algoritmos aplicados con percepción remota puede presentar un reto sino están debidamente calibrados (García et al., 2014).

En López, Plata y Aguilar (2013), se generan una serie de variables para identificar zonas vulnerables a desertificación; sin embargo, se descarta la calibración de los mismos. Una variable utilizada es el contenido de humedad en el suelo utilizando el TVDI (TemperatureVegetation Dryness Index), cuyos resultados se observaron que los valores se comportaban acorde a lo esperado en la distribución del área de estudio, pero sin analizar los datos a fondo.

La propuesta de este trabajo es calibrar los valores de los pixeles acorde a la realidad, para ello se formula un valor de ajuste cambiando los valores de los cuerpos de agua a la interpretación del TVDI de un suelo saturado de humedad (valores en píxeles de 0). Posteriormente, se analizan sus valores resultantes en diferentes usos de suelo y vegetación, de tal manera que los valores se ajusten a las diferentes categorías. 
Por otro lado, se espera tener un parámetro anual calibrado para ingresarlo al modelo de desertificación presentado en López et al. (2013).

\section{Temperature-Vegetation Dryness Index}

Varios estudios para el monitoreo de humedad de suelo han utilizado el índice de sequedad temperatura-vegetación (TVDI - Temperature Vegetation Dryness Index) (Zhenbo, Xuezheng,Warner, Yunjian, Dongsheng, Shaoxiang, y Hongjie, 2008; Yang, Wu, Shi, y Yan, 2008; Han et al., 2010; Chen et al., 2011; Gao, Gao, y Chang, 2011; Holzman, 2012).

El TVDI consiste en un cálculo basado entre el índice de vegetación de diferencia normalizada (NVDI) y la temperatura superficial terrestre (LST) propuesto por Sandholt y otros (2002). El índice puede ser calculado sin la necesidad de información auxiliar (datos de campo); sin embargo, no es bien definido en grandes áreas, si bien, la validación de la observación del TVDI puede volverse dificultosa. El índice se encuentra inversamente correlacionado con la humedad del suelo en la realidad, su ecuación es (Sandholt, Rasmussen y Andersen, 2002; Yang et al., 2008; Pertovt et al., 2008; Chen et al., 2011):

$$
T V D I=\frac{L S T-L S T_{\min }}{a+b * N V D I-L S T_{\min }}
$$

Dónde: LST es el valor de la temperatura superficial que se encuentra en el pixel observado; NVDI es el índice de vegetación de diferencia normalizada; a y b son parámetros calculados a partir de la relación existente entre LST y NVDI obtenido de la regresión lineal; y $\mathrm{LST}_{\min }$ representa la unidad mínima de temperatura de la superficie de la imagen. En este trabajo se utilizó el índice EVI (índice de vegetación mejorado). Acorde con Holzman (2012), el EVI presenta ciertas ventajas respecto al NVDI tales como la consideración del efecto atmosférico y la reducción de la saturación por vegetación muy vigorosa, que en consecuencia se modificó como dato de entrada el NVDI por el EVI.

\section{Materiales y métodos}

\section{Área de estudio}

El Estado de Sinaloa se encuentra ubicado en la parte noroeste de México, colinda con el Estado de Sonora al Norte, Chihuahua y Durango al este, al sur con el Estado de Nayarit y al oeste con el Océano Pacífico. Contiene una superficie total de 5732962 hectáreas, representando el $2.9 \%$ de la superficie del país. Sinaloa se encuentra ubicado a $27^{\circ} 07^{\prime}$ y $22^{\circ} 29^{\prime}$ de latitud norte y $105^{\circ} 23^{\prime}$ y $109^{\circ} 25^{\prime}$ de longitud oeste. La temperatura media anual es en promedio de $24.86^{\circ} \mathrm{C}$, con precipitaciones acumuladas de 674.44 milímetros anuales. Dispone de 19 corrientes y siete cuerpos de agua. Los principales tipos de vegetación son: pastizales, bosques, selvas, matorrales, agricultura, entre otros. El clima varía desde muy seco muy cálido y cálido a templado subhúmedo con lluvias en verano (Inegi, 2012).

\section{Datos}

Se utilizaron como insumo datos de los productos MOD11A2 y MOD13Q1 del sensor MODIS para generar el mapa de sequedad del TVDI. El producto MOD11A2 contiene información de la temperatura superficial terrestre a una resolución espacial de 1000 metros y una temporal de 8 días. El producto MOD13Q1 entre sus datos contiene dos índices de vegetación (NDVI y EVI), cuyas resoluciones son de 250 metros y 16 días. 
Para generar el ajuste del modelo se utilizó el archivo vectorial de cuerpos de agua y la delimitación del área de estudio un archivo de divisiones políticas proporcionado por el portal del Instituto Nacional de Estadística y Geográfica (Inegi).

Para el análisis del ajuste se utilizó el producto MCD12Q1 que contiene los tipos de coberturas, con una resolución espacial de 500 metros y una temporal de un año.

\section{Procesamiento}

En la Figura 1 se muestra el algoritmo seguido en el procesamiento de los datos. Se descargaron todas las imágenes dentro del intervalo en los años de estudio (2003, 2006, 2009 y 2012).

En la generación de las variables anuales (índice de vegetación y temperatura superficial), se utilizó el álgebra de mapas obteniendo una media cuantitativa que represente cada año para ambos insumos. En el caso de la temperatura, se eliminaron aquellas imágenes donde carecían de información (época de lluvias).

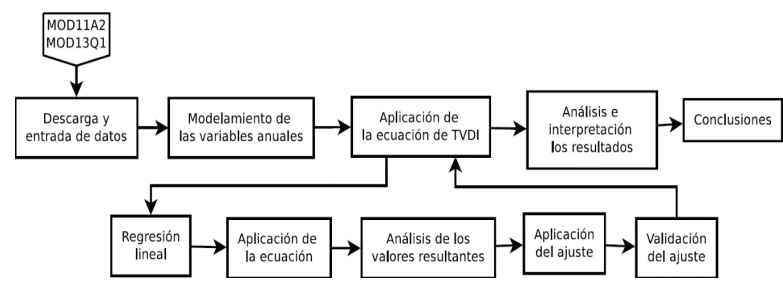

Figura 1. Metodología general del procesamiento. Fuente: Elaboración propia.

Al obtener una variable anual, se procedió a homogenizar las resoluciones espaciales a 250 metros aproximadamente (resolución de las imágenes EVI). En el caso de los archivos vectoriales, se rasterizó y proyectó al sistema de referencia que tienen los productos Modis (sinusoidal) homogenizando las resoluciones a los insumos.
Se generó dos máscaras a partir de los archivos vectoriales, la máscara de Sinaloa que define toda el área de estudio basado solo en la superficie terrestre sin contar las áreas de islas, bahías y mar. La segunda máscara denominada agua contiene solo los cuerpos de agua perenes (presas y diques) que servirá para ajustar el modelo.

La aplicación de la fórmula requiere que se realice una regresión lineal entre el índice y la temperatura para obtener los coeficientes a y b, además, de la obtención de la temperatura mínima dentro del área de estudio para cada año. La temperatura mínima se adquirió de la revisión del histograma de la imagen térmica.

Con los valores de a, by temperatura mínima para cada año, se aplicó la ecuación obteniendo de esta manera un mapa de contenido de humedad, donde los valores representan porcentajes de sequedad en el suelo.

Para realizar el ajuste, se utilizó la máscara de agua para extraer los valores estadísticos del TVDI, bajo la hipótesis que los pixeles asignados como cuerpos de agua se encuentran totalmente saturados. El ajuste fue:

$$
V a=t v d i(x, y)-\left(\mu_{a}-S_{a}\right)
$$

Dónde: $\mathrm{V} a$ es el valor de ajuste, $(\mathrm{x}, \mathrm{y})$ es la imagen del TVDI, $\mu a$ media obtenida de los cuerpos de agua, $S a$ desviación estándar simple. Con el valor de ajuste para cada año, se procede a obtener los mapas de sequedad del suelo aplicando la ecuación:

$$
g(x, y)=\operatorname{TVDI}(x, y)-V a
$$


Dónde: es la imagen del ajuste del modelo, es la imagen obtenida con la ecuación del índice y es el valor ajustado para el año de estudio. De esta manera se obtiene el rango de valores dentro del TVDI (0-1) donde cero representa aquellas áreas saturadas de agua y uno las áreas totalmente secas.

Por último, se analiza el contenido de humedad del suelo ajustado en cada una de las categorías de uso de suelo y vegetación en cada año respectivo utilizando el producto MCD12Q1. Este producto proporciona cinco sistemas de clasificación con diferentes categorizaciones de uso de suelo y vegetación. Para este estudio se seleccionó el esquema categórico proporcionado por la Universidad de Maryland. Las imágenes al final del procesado se transformaron a una proyección local para su visualización (UTM-13N).

\section{Resultados}

\section{TVDI ajustado}

El valor mínimo de la temperatura superficial terrestre, los coeficientes obtenidos a partir de la regresión lineal y los valores de ajuste se muestra en la Tabla 1. El valor mínimo de temperatura está basada en el histograma de cada una de las imágenes. El comportamiento térmico del suelo para cada año se encontró variación entre los años, mostrando que el valor mínimo de temperatura para el año 2003 de 285 ${ }^{\circ} \mathrm{K}, 2006$ de $284{ }^{\circ} \mathrm{K}, 2009$ de $286^{\circ} \mathrm{K}$ y 2012 de $283^{\circ} \mathrm{K}$.

El valor de ajuste en los años 2003, 2006, 2009 dispone de un comportamiento similar. En cuanto al año 2012, no existió demasiada variación entre la media y la desviación estándar en comparación de los otros años, es decir fue el año con menor calibración.
Tabla 1. Valores de coeficiente a y b, temperatura mínima en la imagen y valores de ajuste.

\begin{tabular}{ccccc}
\hline Año & Coeficiente a & Coeficiente b & Valor mínimo $\left({ }^{\circ} \mathbf{F}\right)$ & Valor de ajuste \\
\hline 2003 & 307.277 .678 & -5.284 .441 & 285 & 0.5211 \\
2006 & 29.999 .207 & -1.347 .247 & 284 & 0.5158 \\
2009 & 305.518 .296 & -3.785 .163 & 286 & 0.5195 \\
2012 & 307.482 .835 & -595.071 & 283 & 0.4085 \\
\hline
\end{tabular}

Fuente: Elaboración propia.

Con la calibración de los valores de las imágenes, se procedió a discretizar los valores en 5 categorías ordinales cuyos rangos son equitativos acorde al rango que proporciona el TVDI $(0-1)$ : extremadamente húmedo (0.0 - 0.2), altamente húmedo (0.2 - 0.4), medianamente húmedo $(0.4-0.6)$, bajamente húmedo $(0.6-0.8)$ y sequedad $(0.8-1.0)$.

En la Figura 2, se muestran los porcentajes que cubren las diferentes categorías de humedad en el territorio, mostrando que gran parte una situación medianamente húmedo, debido a la variación existente entre los valores de entrada.

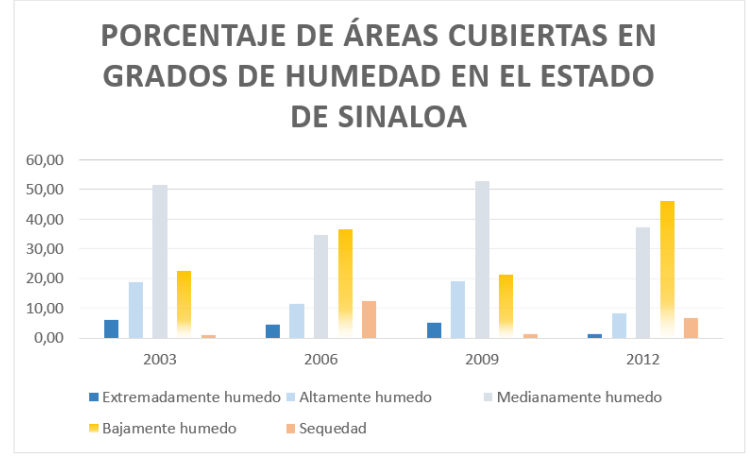

Figura 2. Porcentaje de áreas cubiertas en grados de humedad en el Estado de Sinaloa, México. Fuente: Elaboración propia.

En la figura 3 se muestran los cuatro mapas resultantes de la calibración. En los años 2003 y 2009, tienen un comportamiento similar, la categoría de mayor predominancia es 
medianamente húmedo con porcentajes de $51.64 \%$ y $53.01 \%$, respectivamente, seguidos de bajamente húmedo con $22.72 \%$ para el año 2003 y $21.38 \%$ para el año 2006 , continuando con altamente húmedo (18.71\% y 19.04\%), seguidos por los extremos de extremadamente húmedo $(4.61 \%$ y $5.24 \%)$ y sequedad $(0.88 \%$ y $1.33 \%)$.

Por otro lado, en el año 2006, aumenta considerablemente las zonas con sequedad $(12.43 \%)$, y las zonas bajas de humedad $(36.68 \%)$, disminuyendo las zonas medianamente humedad $(34.80 \%)$, altamente húmedas (11.48\%), y extremadamente húmedas (4.61\%) en comparación del año 2003. En el año 2012, el comportamiento se presenta de manera similar al año 2006, pero el año 2012 representa el año más seco entre los años de estudios tenido como resultado las zonas clasificadas como bajamente húmedo representan el $46.05 \%$ del territorio, seguidos de medianamente húmedo (37.32\%), altamente húmedo (8.44\%), zonas secas $(6.71 \%)$ y extremadamente secas $(1.48 \%)$. Las zonas más secas se presentan en la parte central del Estado de Norte a Sur; por otro lado, las zonas con más humedad se encuentran en las zonas serranas.

\section{Uso de suelo y vegetación}

Del producto MCD12Q1 del esquema de la Universidad de Maryland, se utilizaron 14 categorías. Los valores estadísticos para cada categoría se muestran en la Tabla 2, mostrando valores porcentuales de sequedad del suelo.

Al aplicar la ecuación de TVDI sin ajustar, el valor mínimo de las medias para los años 2003 y 2006 se presenta en los Bosques Perennes de Latifoliadas con un valor medio de 0.6257 y 0.6274 para ambos años. Para los caso de los año 2009 y 2012, los valores mínimos son en los cuerpos de agua ( 0.6826 y 0.6447 respectivamente).

Con la aplicación de la ecuación de ajuste, los cuerpos de agua se representaron con el valor de 0 dentro de los primeros decimales en todos los años con ciertas variaciones en las diezmilésimas.

Analizando los valores porcentuales de sequedad generados por la ecuación de ajuste en cada categoría de uso de suelo y vegetación, en los cuatro años de estudio los que contienen un mayor margen de humedad en el suelo son los bosques perennes de latifolias seguido de las coníferas. Las áreas urbanas y los suelos desnudos o con escasa vegetación representan las áreas más secas con excepción del año 2006, donde el área más seca lo representó en las Sabanas (figura 4).

El año 2009 represento ser un año húmedo en todos los uso de suelo y vegetación. Por el otro lado, el año 2012 represento ser un año seco, seguido de los años 2006 y 2003.

En el año 2003, las categorías con cierto grado de sequedad se presentaron en los suelos desnudos o con escasa vegetación (56\%), áreas urbanas (55\%) y pastizales (51\%). En el año 2006, áreas urbanas y sabanas con $61 \%$ de sequedad en el suelo, seguido de matorral abierto $(60 \%)$. En el año 2009, se mostró un mayor grado de sequedad en los suelos desnudos de $45 \%$, seguido de áreas urbanas y sabanas (43\%). En el año 2012, se presentó los porcentajes más altos de sequedad, suelos desnudos con $67 \%$, áreas urbanas y matorrales con $65 \%$ y $64 \%$, respectivamente.

Por otro lado, en los años de estudios la categoría de bosques perennes de coníferas y de latifoliadas presentan las categorías con menor grado de sequedad. 

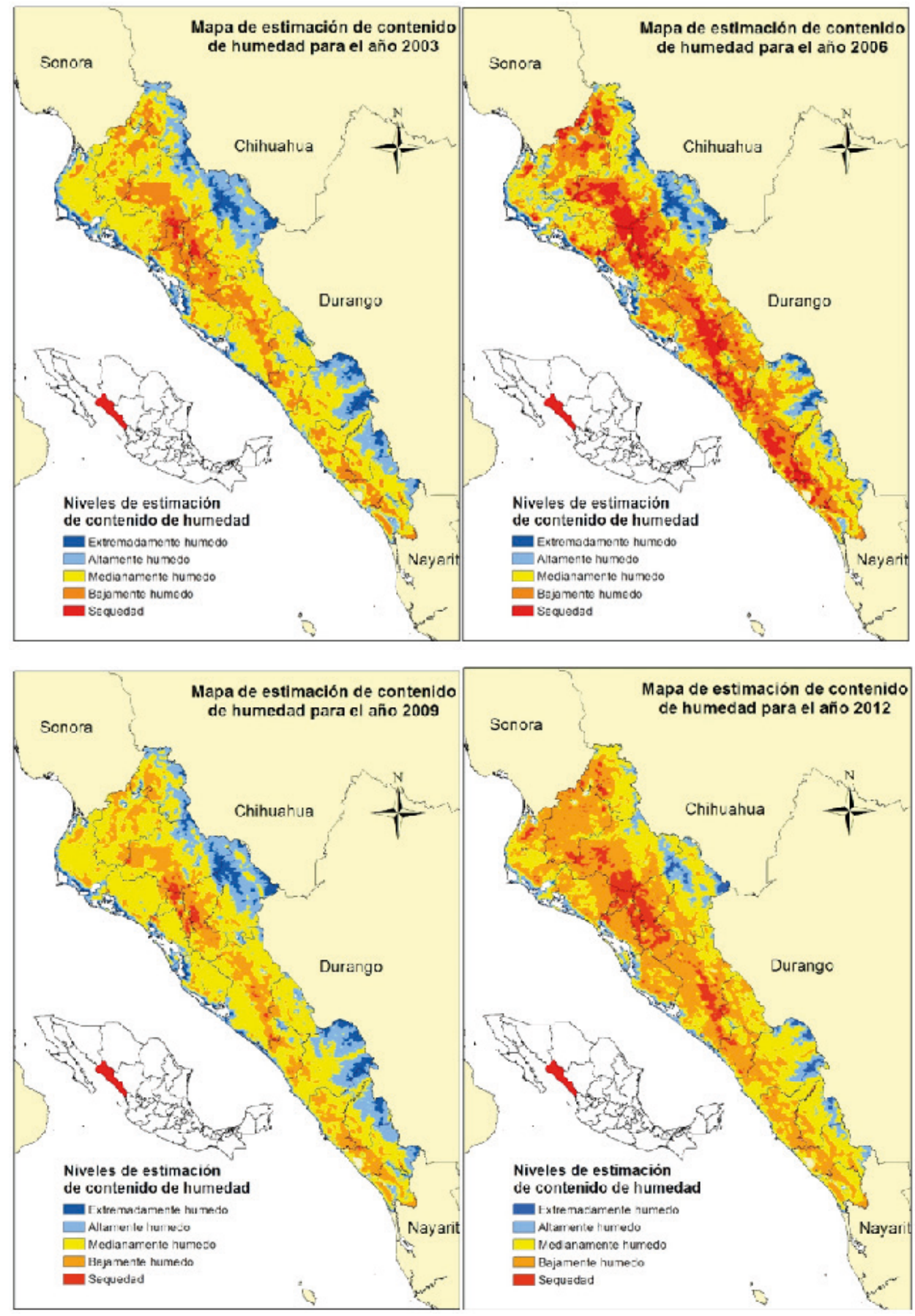

Figura 3. Mapas de estimación de contenido de humedad resultante del TVDI en los años 2003, 2006, 2009 y 2012 Sinaloa, México. Fuente: Elaboración propia. 
Tabla 2. Valores porcentuales de sequedad del suelo antes y después del ajuste.

\begin{tabular}{|c|c|c|c|c|c|c|c|c|c|c|c|c|}
\hline \multirow{2}{*}{$\begin{array}{l}\text { Uso de suelo y vegetación (MODIS) } \\
\text { Media. } \\
\sigma: \text { Desviación estándar. } \\
\text { A: Media después del valor de ajuste } \\
\text { ND: Sin dato }\end{array}$} & \multicolumn{3}{|c|}{2003} & \multicolumn{3}{|c|}{2006} & \multicolumn{3}{|c|}{2009} & \multicolumn{3}{|c|}{2012} \\
\hline & $\mu$ & $\sigma$ & A & $\mu$ & $\sigma$ & A & $\mu$ & $\sigma$ & A & $\mu$ & $\sigma$ & A \\
\hline Cuerpo de agua & 0.65 & 0.13 & 0.00 & 0.69 & 0.18 & 0.00 & 0.68 & 0.16 & 0.00 & 0.64 & 0.23 & 0.00 \\
\hline Bosque Perenne de Coniferas & 0.71 & 0.10 & 0.18 & 0.71 & 0.16 & 0.20 & 0.76 & 0.14 & 0.14 & 0.77 & 0.13 & 0.36 \\
\hline Bosque Perenne de Latifolada & 0.62 & 0.12 & 0.10 & 0.62 & 0.07 & 0.11 & 0.71 & 0.15 & 0.09 & 0.66 & 0.09 & 0.25 \\
\hline Bosque Deciduo de Coeiferas & 0.83 & 0.12 & 0.31 & 0.81 & 0.01 & 0.29 & $\mathrm{ND}$ & $\mathrm{ND}$ & ND & 0.86 & 0.06 & 0.45 \\
\hline Bosque Decidao de Latifoladas & 0.98 & 0.10 & 0.46 & 1.09 & 0.11 & 0.58 & 0.97 & 0.09 & 0.35 & 0.97 & 0.08 & 0.56 \\
\hline Bosques Mixtos & 0.86 & 0.22 & 0.34 & 0.89 & 0.22 & 0.37 & 0.81 & 0.18 & 0.19 & 0.87 & 0.15 & 0.46 \\
\hline Matorral Cerrado & 1.00 & 0.18 & 0.48 & 0.95 & 0.19 & 0.43 & 0.89 & 0.15 & 0.27 & 0.94 & 0.17 & 0.54 \\
\hline Matorral Abierto & 1.03 & 0.14 & 0.51 & 1.11 & 0.18 & 0.60 & 1.04 & 0.15 & 0.42 & 1.05 & 0.15 & 0.64 \\
\hline Savarnas & 0.99 & 0.15 & 0.47 & 1.13 & 0.19 & 0.61 & 1.05 & 0.17 & 0.43 & 1.02 & 0.14 & 0.61 \\
\hline Pastos & 1.03 & 0.16 & 0.51 & 1.10 & 0.26 & 0.58 & 1.00 & 0.16 & 0.38 & 1.03 & 0.14 & 0.62 \\
\hline Cultivos & 0.97 & 0.18 & 0.45 & 1.09 & 0.21 & 0.58 & 1.02 & 0.18 & 0.40 & 0.96 & 0.19 & 0.55 \\
\hline Áreas Uribanas y Construcciones & 1.07 & 0.13 & 0.55 & 1.12 & 0.17 & 0.61 & 1.05 & 0.11 & 0.43 & 1.06 & 0.12 & 0.65 \\
\hline Suelos Destuudo o con Escasa Vegetaciön & 1.08 & 0.09 & 0.56 & 1.10 & 0.12 & 0.59 & 1.06 & 0.09 & 0.45 & 1.08 & 0.09 & 0.67 \\
\hline No Clasticado & 0.78 & 0.15 & 0.26 & 0.83 & 0.20 & 0.31 & 0.79 & 0.15 & 0.17 & 0.81 & 0.18 & 0.40 \\
\hline
\end{tabular}

Fuente: Elaboración propia.

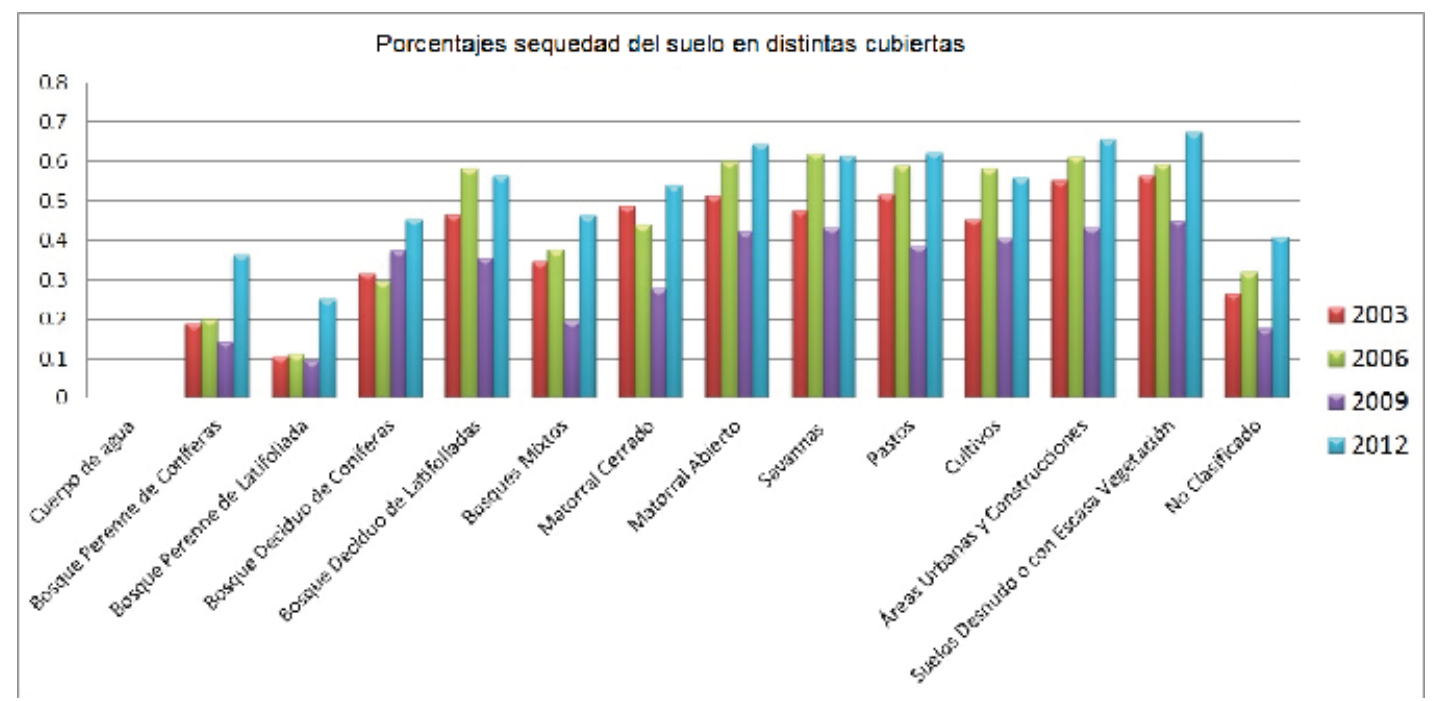

Figura 4. Gráfica del comportamiento de la sequedad en el suelo en los años de estudio (0 totalmente saturado de agua y 1 totalmente seco). Fuente: Elaboración propia. 


\section{Discusión de resultados}

Los resultados muestran el comportamiento esperado en base del ajuste. El TVDI ha sido utilizado de diferentes maneras. En nuestro caso es la generación de una variable anual de entrada a un modelo de desertificación para el Estado de Sinaloa, México. Por lo que se buscó una manera de calibrar los datos de los pixeles acorde a una realidad de interpretación, es decir, valores de 0 representan suelos saturados de agua y valores de 1 son suelos totalmente secos.

De acuerdo con Ravelo, Sanz, y Douriet (2014), el periodo 2011-2012, se consideran como los años más severos en términos de sequías en la última década para el Estado de Sinaloa. Confirmando la variación de sequedad presentada en el año 2012 en nuestros resultados.

Existen diversos trabajos que han utilizado el TVDI, por ejemplo, Zhenbo et al. (2008), utilizaron el TVDI para determinar la relación del contenido de humedad del suelo con respecto a la migración de la plaga de langosta migratoria (Locusta migratoria manilensis), determinaron la precisión de los resultados generados por el índice en 12 muestreos de suelo, seleccionando 3 áreas pilotos como puntos de observación del TVDI, cuyos resultados mostraron tener una correlación negativa significativa acorde a la humedad del suelo. Por otro lado, Chen et al. (2012), compararon los resultados con la precipitación, indicando que el TVDI es generalmente sensible a la precipitación en la mayoría de los casos, pero los valores del TVDI durante o después de la precipitación mostraron resultados que indicaban menor contenido de humedad. Prevort et al. (2008), mencionan que valores mayores a 1 (que se considera un límite seco acorde al TVDI), fueron detectados como umbrales naturales de estrés hídrico. Además, los bosques no son sensibles a las variaciones de la precipitación que puedan presentarse en las diferentes épocas del año, por lo que estas zonas se observan valores bajos de TVDI a lo largo del año. Holzman (2012), menciona que existe una gran relación entre el índice y el contenido volumétrico existente en el suelo dentro del rango de 10 a $20 \mathrm{~cm}$ de profundidad en campos agrícolas.

\section{Conclusiones}

La aplicación del TVDI requiere de un área que permita definir los valores de la regresión lineal para la ecuación entre la temperatura e índice de vegetación. Sin embargo, el resultado puede generar valores que salgan fuera del rango de la interpretación o aquellas zonas que se conoce de ante mano que el suelo se encuentra totalmente saturado de agua y en el índice, la interpretación muestra que existe cierto grado de sequedad, generando cierto grado de error. Por lo que presentó una manera de ajustar los valores de los píxeles a partir de un simple ajuste con estadística descriptiva.

La estimación proporcionada por el TVDI ajustado tiene un comportamiento esperado dentro de las coberturas de uso de suelo y vegetación. Sin embargo, al ser un análisis anual, existe cierto grado de variación, por ejemplo, la categoría de cultivos (zonas agrícolas), durante el ciclo vegetativo se mantiene al suelo con cierto grado de humedad fácilmente aprovechable por las plantas para su desarrollo pero este no se ve reflejado en el índice TVDI debido a que durante la siembra y crecimiento de los cultivos, el índice de vegetación refleja gran parte de su información de un suelo descubierto aunque el suelo pueda existir gran parte de humedad. En consecuencia, al ser una media anual esto afecta en cierta medida la estimación para suelos agrícolas como variable de entrada anual en esta categoría. 
Respecto al objetivo de generar una variable de entrada para un modelo específico, este procedimiento permitió distribuir los valores de manera rápida acorde al contenido de humedad de los cuerpos de agua, obtenido así un parámetro de entrada más calibrado.

La validación del ajuste conforme a los usos de suelo y vegetación muestra que la humedad se distribuye de manera correcta en las distintas categorías, lo que permite dar una mayor robustez al modelo ajustado.

\section{Referencias bibliográficas}

Chen, C., Chen, C., Son, N., \& Chang, L. (2011). Monitoring of soil moisture variability in relation to rice cropping systems in the vietnamese mekong delta using Modis data. Applied Geography, 31(2), 463-475.

Chen, C.F, Lin, Y.J., Chang, L.Y. \& Son, N.T. (2012). Retreving surface soil mositure from MODIS and AMSR-E data: a case of study in Taiwan. International Archives of the Photogrammetry, Remote Sensing and Spatial Information Sciencies (39). $379-383$.

CONAGUA. (2011). Estadística del agua en México. Conagua: Comisión Nacional del Agua.

Gao, Z, Gao, W, \& Chang, N.-B. (2011). Integrating temperature vegetation dryness index (TVDI) and regional water stress index (RWSI) for drougth assessment with the aid of LANDSAT $\mathrm{TM} / \mathrm{ETM}+$ images. International Journal of applied earth observation and geoinformation(13), 495 - 503.
García, M, Fernández, N, Villagarcía, L, Domingo, F, Puigdefábregas, J, \& Sandholt, I. (2014). Accuary of temperature-vegetation dryness index using MODIS under water-limited vs energy-limited evapotranspiration conditions. Remote Sensing of Enviroment(149), 100-117.

Han, Y, Wang, Y \& Zhao, Y. (2010). Estimating soil moisture conditions of the Greater Changbai Mountains by land surface temperature and NVDI. IEEE Transactions on geoscience and remote sensing, 48(6), 2509 - 2512.

Holzman, M. E. (2012). Estimación del estrés hídrico en cultivos y su relación con rendimientos en la región pampeana mediante imágenes de temperatura de superficie e índice de vegetación. Tesis Doctoral. Universidad Nacional del Sur

Inegi. (2012). Anuario estadístico de Sinaloa 2012. INEGI: Instituto Nacional de Estadística y Geografía.

IPCC. (2008). Cambio climático 2007: informe de síntesis. Contribución de los grupos de trabajo I, II y III al Cuarto Informe de evaluación del Grupo Intergubernamental de Expertos sobre el Cambio Climático.

López Beltrán, M. A, Plata, W, Aguilar J \& Guzmán Galindo, T. (2013). Generación de variables geoespaciales para determinar zonas propensas a desertificación. Memorias del congreso "XX Reunión Nacional Selper-México, San Luis Potosí, 2013". 
Pertovt, L, Rivas, R., Juliano, S, Osmar, Yang, X., Wu, J., Shi, P., \& Yan, F. (2008). W \& Vives, L. (2008). Análisis de condicionantes ambientales del estrés hídrico de la vegetación en el sur de Brazil mediante imágenes NOAA-AVHRR. Boletín Geológico y Minero, 119(1), 119-124.

Ravelo, A, Sanz Ramos, R, \& Douriet Cárdenas, J. (2014). Detección, evaluación y pronóstico de las sequías en la región del Organismo de Cuenca Pacífico Norte, México. Agriscientia, 31(1), 11 - 24.

Sandholt, I, Rasmussen, K \& Andersen, J. (2002). A simple interpretation of the surface temperature/vegetation index space for assessment of surface Modified triangle method to estimate soil moisture status with moderate resolution imaging espectroradiometer Modis products. The international archives of the phogrammetry, remote sensing and spatial information sciences, 37, pp. 555-560.

Zhenbo, L, Xuezheng, S, Warner, E., Yunjian, G, Dongsheng, Y, Shaoxiang, N \& Hongjie, W. (2008). Relationship between oriental migratory locust plague and soil mositure extracted from MODIS data. International Journal of Applied Earth Observation and Geoinformation(10), 84 - 91. Enviroment(79), 213 - 224. 
